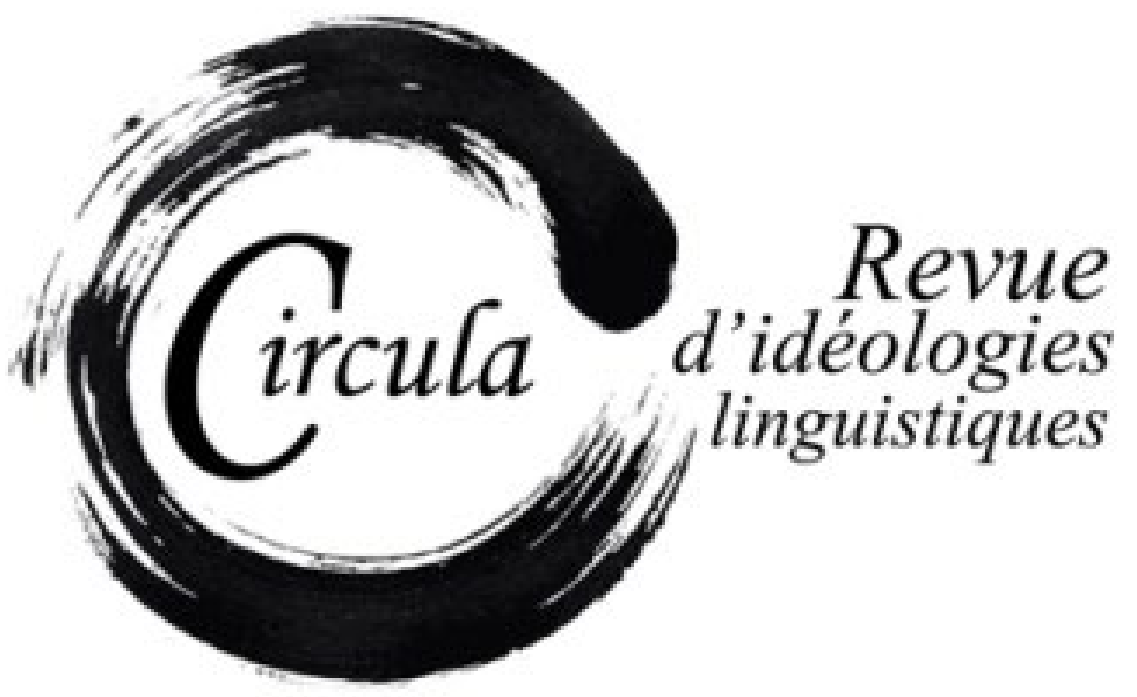

TITRE: LA CLARTÉ COMME PRINCIPE DIRECTEUR DANS LE DISCOURS NORMATIF SUR LES USAGES COMMUNICATIONNELS EN PRESSE ÉCRITE QUÉBÉCOISE

Auteur(s): Franz Meier, Universität Augsburg

Revue: CirCula, NUMÉRO 4

PAgES: $87-105$

ISSN: 2369-6761

DiRecteurs: Wim Remysen et SABINE SCHWARZE

URI: HTTP://HDL.HANDLE.NET/11143/10178

DOI: $10.17118 / 11143 / 10178$ 


\section{La clarté comme principe directeur dans le discours normatif sur les usages communicationnels en presse écrite québécoise}

Franz Meier, Universität Augsburg franz.meier@philhist.uni-augsburg.de

Résumé : Les journalistes de la presse écrite quotidienne sont obligés d'écrire leurs articles de manière à ce qu'ils soient facilement compréhensibles par le plus grand nombre de lecteurs possible. À cette fin, la valeur de clarté est souvent présentée comme un principe rédactionnel important qui devrait assurer l'accessibilité de l'écriture journalistique. Dans cette optique, nous nous intéresserons aux conceptions de la langue que les professionnels des médias écrits québécois attribuent à la notion de clarté, idéologie linguistique qui traverse le discours sur la langue française depuis plusieurs siècles. Nous traiterons cet aspect à partir d'un corpus d'entretiens semi-dirigés réalisés auprès de seize enseignants d'écriture journalistique et de douze chroniqueurs. Nous analyserons dans quelle mesure les propos des témoins correspondent au discours normatif véhiculé dans les manuels de journalisme québécois.

Mots-clés : clarté ; normes communicationnelles; journalisme de communication ; presse écrite québécoise

Abstract: Daily newspapers journalists have to write their articles in a way that they can be easily understood by the greatest number possible of readers. To this end, the notion of clarity is often presented as an important editorial principle that should guarantee the accessibility of journalistic writing. This article focuses on the language conceptions to which the professionals of the Quebec written media attribute the term clarity, a linguistic ideology that has run through the discourse on the French language for several centuries. Our analysis is based on a data collection of semi-structured interviews with sixteen teachers of journalistic writing and twelve columnists. The article analyses the extent to which the participants' statements correspond to the normative discourse conveyed in the journalism textbooks published in Quebec.

Keywords: clarity; communicational norms; communication journalism; Quebec daily newspapers 


\section{Introduction}

Les journalistes des médias écrits sont généralement amenés à adapter les usages qu'ils font de la langue de manière à ce qu'ils puissent établir et maintenir le contact avec leurs lecteurs, communiquer avec eux. Dans le discours normatif sur ces usages communicationnels, la clarté est constamment présentée comme un impératif rédactionnel qui devrait régir les pratiques des journalistes. Dans cet article, nous nous proposons d'exposer la façon dont les professionnels des médias écrits québécois conçoivent ce qu'est une écriture journalistique dite claire et dans quelle mesure leurs conceptions divergent des règles qu'on trouve dans les manuels de journalisme publiés au Québec.

\section{Les journalistes comme communicateurs dans les médias de masse}

Les journalistes de la presse écrite produisent leurs textes dans une situation de communication particulière, celle des médias de masse, où les échanges entre les partenaires se caractérisent par une relation «non-dialogique » (Charaudeau, 1988 : 76), parfois aussi nommée mediated quasi-interaction (Thompson, 1995 : 84). Dans cette situation de communication, les lecteurs n'ont pas la possibilité d'intervenir dans la rédaction des articles et les journalistes n'ont pas d'accès immédiat aux réactions du lectorat par rapport à leurs textes (Burger, 2005). Même si les journaux mettent en place plusieurs outils pour permettre aux lecteurs de s'exprimer, par exemple des courriers (Burger, 2005), et que les nouveaux moyens de communication en ligne, comme les blogues et le clavardage, confèrent progressivement une « dimension interactive, voire participative » (Grevisse, 2008 : 214) aux médias de masse, leur public demeure un ensemble hétéroclite de personnes plus ou moins anonymes et largement dispersées dans le temps et dans l'espace (Bell, 1991 ; Fairclough, 1995 ; Lebsanft, 2001).

C'est ainsi que les journalistes ne peuvent se faire qu'une image prototypique de leur lectorat cible, dont ils sont certes, du moins en partie, disjoints, mais auquel ils doivent ajuster le traitement des messages à fournir (Bell, 1991 ; Burger, 2000). Cet alignement s'avère d'autant plus important dans le paradigme du journalisme de communication'슬 où les professionnels des médias sont tenus d'établir « des liens de connivence et d'intersubjectivité » (Brin, Charron et de Bonville, 2004 : 4) avec un public imaginaire, c'est-à-dire qu'ils sont obligés de présenter les informations à transmettre de sorte qu'elles soient le plus compréhensibles à un maximum de lecteurs (Fairclough, 1995). Les journalistes ne sont donc plus de simples diffuseurs de contenus informatifs, mais ils exercent le rôle d'« expert[s] en matière de formes efficaces » (Lavoinne, 1991 : 171) qui, dans un contexte d'im-

1. Brin, Charron et de Bonville (2004) divisent l'histoire du journalisme québécois, qu'ils situent plus largement dans le contexte du journalisme nord-américain, en quatre périodes qui s'enchainent et qui correspondent à quatre façons particulières de concevoir les pratiques journalistiques. Ils distinguent alors le journalisme de transformation qui, au début du XIX siècle, cède la place au journalisme d'opinion qui, à son tour, est remplacé entre 1880 et 1910 par le journalisme d'information, auquel succède le journalisme de communication, qui apparait à partir des années 1970 et 1980. 
portante concurrence sur le marché des médias, savent traiter des données « de manière à créer l'illusion d'une communication interpersonnelle avec le public » (Brin, Charron et de Bonville, 2004 : 9). Autrement dit, dans le journalisme de communication, c'est notamment la fonction phatique, assurant le lien entre journalistes et lecteurs, qui est mise de l'avant, et moins la fonction référentielle, plus axée sur le contenu du message (Brin, Charron et de Bonville, 2004). Compte tenu du caractère de plus en plus participatif des médias de masse, Grevisse conclut que les journalistes doivent plus que jamais considérer les lecteurs comme des « partenaire[s] de la communication de l'information [qui] prennent des places très variables dans la production de l'information » (Grevisse, 2008 : 214).

\section{La clarté comme principe directeur des normes communication- nelles}

Pour expliquer comment la situation de communication des médias de masse influe sur les usages des journalistes, nous recourons au concept de normes communicationnelles, proposé initialement par Houdebine (1983) et développé ensuite par Houdebine et Baudelot (1985). Ces normes « se caractérise[nt] essentiellement par la prise en compte du destinataire, même s'il s'agit d'un destinataire idéalisé ou fantasmé » (Houdebine et Baudelot, 1985: 62) et commandent un emploi strictement utilitaire et instrumental des ressources linguistiques, ce qui peut même aller à l'encontre des normes prescriptives². Dans un but de «facilité de compréhension entre les locuteurs» (Houdebine-Gravaud, 2002 : 15), les normes communicationnelles obligent donc l'émetteur de messages à livrer les informations en fonction du profil et des besoins communicationnels des destinataires.

Selon Houdebine (1988: 140), le souci d'être compris par le plus grand nombre de lecteurs relève d'une conception de la langue qui se définit surtout par le principe de clarté. Ainsi, « savoir écrire pour un journaliste c'est [...] "écrire [de façon] claire et compréhensible", "écrire [de façon] claire et simple" » (Houdebine et Baudelot, 1985 : 63). Houdebine constate à juste titre qu' " on retrouve là de vieux arguments [qui] sont utilisés de façon nouvelle» (Houdebine, 1988: 140). En effet, l'idéologie de la clarté revient régulièrement dans le discours sur la langue en France depuis le XVIIe siècle (Ludwig et Schwarze, 2012; Paveau et Rosier, 2008 ; Weinrich, 1961; Yaguello, 2008), où elle est non seulement considérée comme une propriété de l'identité culturelle des Français, mais aussi comme une caractéristique de leur «façon (nationale) de parler et d'écrire» (Swiggers, 1987 : 14). Dans cette optique,

2. À ce propos, Houdebine soutient que « [la] préférence est alors donnée par les journalistes au compréhensible, au communicable, cela même à l'écrit et avec méconnaissance ou connaissance de la norme prescriptive » (Houdebine-Gravaud, 2002: 15). À titre d'exemple, l'auteure mentionne l'expression mettre à jour pour mettre au jour ou une rue passagère pour une rue passante ou le verbe pallier à pour pallier (voir Houdebine-Gravaud, 2002). 
la présumée clarté du français est parfois même vue comme « un épiphénomène de la clarté des Français : à langue logique, locuteurs logiques»(Swiggers, $2010: 451)^{3}$.

Envisagée comme un principe directeur des normes communicationnelles, la clarté impose une série de choix linguistiques qui forment un style dit fonctionnel propre au domaine journalistique (Eroms, 2008 ; Sanders, 1977), qui est généralement décrit sous forme de règles brèves dans la plupart des manuels de journalisme, y compris dans ceux publiés au Québec, où on trouve par exemple l'énoncé de principe selon lequel « la clarté doit être la marque de toute écriture journalistique » (Maltais, 2010 : 18 ; voir aussi Noël, 2009 ; Ross, 2005 ; Sormany, 2011). Compte tenu de la grande importance accordée à la clarté dans le discours normatif sur les pratiques journalistiques, nous cherchons à savoir la place qu'occupe la notion dans l'imaginaire linguistique des professionnels des médias écrits québécois et les conceptions de la langue qui y sont attachées.

\section{Les composantes de la clarté journalistique}

Nous avons mené notre analyse à partir d'un corpus composé de 39 entrevues semi-dirigées conduites entre 2011 et 2013 auprès de 16 enseignants de rédaction journalistique, de 12 chroniqueurs, c'est-à-dire de journalistes qui donnent régulièrement leur opinion sur un sujet d'actualité ou sur un thème de leur choix, et de 11 langagiers, à savoir des traducteurs et des réviseurs qui œuvrent dans le milieu journalistique (Meier, 2016). Notre analyse porte ici exclusivement sur le discours des enseignants, qui travaillent au sein de diverses universités québécoises, et des chroniqueurs, qui écrivent à La Presse, au Devoir et au Soleil dans les sections actualités politiques, questions éthiques et sociales ainsi qu'arts et spectacles ${ }^{4}$. Chaque entrevue contient une vingtaine de questions, dont une concerne spécifiquement la clarté et qui a été retenue dans la présente analyse, à savoir « L'ensemble des guides de rédaction journalistique mentionne la clarté de la langue comme point de repère de l'écriture de presse. En quoi consiste cette clarté pour vous?».

3. Voir aussi Weinrich (1961), qui écrit à ce sujet: « Car les Français ont mis au moins autant de passion à se saisir de l'ethos de la clarté qu'ils ont mis d'empressement à croire au mythe de la clarté. Et cet ethos a effectivement produit en France une mesure de clarté de la pensée qui est aujourd'hui comme depuis toujours un modèle pour les voisins. Cette clarté de la pensée et de l'expression est la véritable clarté française, la clarté des Français » (Weinrich, 1961 : 544 ; nous traduisons).

4. L'étude du discours normatif des enseignants et des chroniqueurs est d'un intérêt particulier puisque ces personnes profitent d'une certaine autorité en matière de langue journalistique. Ainsi, les enseignants sont à la fois les promoteurs et les émetteurs d'impératifs rédactionnels qui transmettent des traditions d'écriture aux futurs journalistes et les chroniqueurs, à leur tour, sont souvent considérés, pour en croire plusieurs manuels (Maltais, 2010 ; Noël, 2009), comme des exemples d'une écriture journalistique particulièrement vivante et imagée. 


\subsection{Clarté et structures syntaxiques}

Tous les répondants reconnaissent le principe de clarté comme une norme à suivre lorsqu'il s'agit d'assurer qu'un article soit bien compris par les lecteurs. En dépit de cette unanimité, la définition que proposent les témoins de la clarté journalistique est loin d'être univoque et repose sur différents types d'arguments. La plupart des répondants mettent en avant des aspects qui concernent la phrase et sa structure syntaxique. Cette clarté phrastique résiderait surtout dans l'emploi de constructions « relativement simples » $(\mathrm{P} 07)^{5}$ qui suivent le mieux possible le modèle canonique de la phrase, c'est-à-dire la phrase de base, parfois appelée aussi phrase élémentaire (Riegel, Pellat et Rioul, 2009). Ainsi, pour les répondants, « des phrases idéales, c'est sujet, verbe, complément » (P20), tandis que « des phrases trop complexes, avec trop de subordonnées, où [...] le sujet est éloigné de son verbe » (P07) doivent être évitées. Cette attitude défavorable envers la subordination revient aussi dans l'ensemble des manuels de journalisme consultés (Maltais, 2010 ; Noël, 2009; Ross, 2005 ; Sormany, 2011) et abonde pendant l'enquête. Par exemple, les enseignants perçoivent généralement la formule sujet, verbe, complément comme un repère normatif fondamental qui occupe une place centrale dans leurs ateliers de rédaction:

ce que je dis à mes étudiants dans les premières semaines, c'est une phrase, c'est un sujet, un verbe, un complément, et essayez pas de faire des phrases trop complexes, commencez par faire des phrases simples, commencez par prendre un verbe qui est actif, puis à mettre le ... le ... celui qui fait action, qui pose l'action comme sujet et éliminez les qui, les que, les dont, éliminez, essayez d'éliminer les subordonnées dans vos phrases parce que lorsque vous faites une phrase trop complexe, de toute façon, elle devient ... elle devient plus difficile à ... à comprendre (P07)

Les témoins attribuent donc une valeur communicative à la phrase canonique, qui, selon eux, permettrait une meilleure compréhension des textes journalistiques par les lecteurs ${ }^{6}$. Cette argumentation figure aussi dans la majorité des guides d'écriture, dont celui de Noël (2009), qui établit même une correspondance entre l'ordre des mots et l'ordre de la pensée, argument puissant que l'on retrouve sous l'appellation d'ordre naturel dans le discours sur la clarté du français depuis le Siècle des lumières (Ludwig et Schwarze, 2012)ㄹ:

5. Dans les exemples cités, les tours de parole des participants ont été marqués par un numéro de code suivant le modèle PXX, où la variable XX indique le numéro de l'entretien. La transcription évite dans la mesure du possible la ponctuation. Les virgules sont néanmoins utilisées pour indiquer la fin d'un segment de la chaine parlée, une pause est indiquée par ... et une séquence omise, par [...].

6. Néanmoins, la forme canonique de la phrase peut aussi causer des ambiguïtés. Swiggers (1987: 17) mentionne par exemple j'ai vu peindre cette femme et il a fait peindre sa femme.

7. Mentionnons notamment Le discours sur l'universalité de la langue françoise (1784) de Rivarol : "Ce qui distingue notre langue des anciennes et des modernes, c'est l'ordre et la construction de la phrase. Cet ordre doit toujours être direct et nécessairement clair. Le français nomme d'abord le sujet de la phrase, ensuite le verbe, qui est l'action, et enfin l'objet de cette action : voilà la logique naturelle à tous les hommes; voilà ce qui constitue le sens commun » (Rivarol, [1784] $1929: 88)$. 
«Les experts en électricité prévoient une flambée des prix du gaz. » C'est ce genre de propositions que les journalistes doivent favoriser. Sujet, verbe, complément : voilà l'ordre logique de la pensée (Noël, 2009: 32).

Les répondants associent aussi la clarté de la phrase à sa longueur et favorisent généralement l'utilisation de « phrases courtes » (P12), où, selon un enseignant, « on essaie d'éviter d'avoir plus que dix-sept mots » (P14), nombre approximatif que l'on trouve parfois aussi dans les manuels (Maltais, 2010 ; Noël, 2009 ; Ross, 2005) ${ }^{8}$. Par conséquent, l'emploi de phrases longues est souvent très mal perçu et cantonné au domaine de la littérature. Tel est le cas dans la citation suivante, où un enseignant érige les phrases de Marcel Proust en anti-modèle de la clarté journalistique :

une règle évidente en journalisme, c'est la phrase courte, la phrase synthétique, concise, mais courte, donc adieu les ... les Proust de ce monde, s'ils veulent être des ... des journalistes, c'est ... c'est pas du tout ... c'est pas du tout le but (P15)

La condamnation des phrases proustiennes constitue, pour en croire Paveau et Rosier (2008), un thème récurrent dans le discours normatif sur la clarté en matière de syntaxe, thème qui, comme le montre l'extrait suivant tiré du manuel de Noël (2009), semble aussi bien reçu dans la tradition de réflexion sur l'écriture journalistique au Québec :

Et à moins d'être un grand écrivain comme Marcel Proust, il est plus facile d'écrire de belles phrases courtes que de belles phrases longues. Elles répondent mieux à deux des quatre exigences du style journalistique : clarté et concision (Noël, 2009 : 36).

Si les enseignants reconnaissent l'importance d'utiliser des phrases courtes et simples, quelquesuns précisent qu' « il faut pas non plus penser que c'est sujet, verbe, complément sans aucune considération, [... . il faut être intéressant » (P12). En ce sens, l'alternance dans la structure et la longueur des phrases contribuerait à une écriture plus vivante, où « la clarté est aussi dans le choix des rythmes [et dans] la musicalité de [la] langue» (P04). De ce point de vue stylistique, l'alignement parfait sur le modèle de la phrase canonique pourrait porter atteinte à l'efficacité communicationnelle des textes, car la répétition d’un seul schéma syntaxique ennuierait les lecteurs:

la clarté, elle est dans la structure de la syntaxe, mais là encore, pas forcément la phrase canonique en français, sujet, verbe, complément, parce qu'un des éléments qui fait la clarté, et bon, le style aussi, c'est le fait qu'il y a un rythme, et le rythme fait souvent ... fait ... il faut pour qu'il y ait du rythme ... qu'il y ait ... en fait, des ruptures et des ruptures syntaxiques notamment, c'est que si on suivait toujours sujet, verbe, complément, sujet, verbe, complément, on arriverait à un ronron, ça endormirait, et donc ça, c'est peut-être plus du style que de la clarté au départ, mais je pense que c'est fondamental de ... d'avoir des phrases qui ... dont la

8. Noël consacre même un chapitre entier à l'importance de « faire des phrases courtes » (Noël, 2009: 21), conseil rédactionnel qu'il justifie par l'indice de lisibilité de Flesh (1949) et de Gunning (1968). 
... la structure est un peu syncopée, alors on a des phrases longues, des phrases courtes, des phrases avec inversion, des phrases avec incise ou pas, et je me porte un peu en faux contre les gens qui disent qu'un style clair et efficace, c'est uniquement des phrases courtes (P11)

Les chroniqueurs, quant à eux, insistent aussi sur la nécessité d'« éviter les longues phrases » (P32), mais ils ajoutent que tous les genres journalistiques ne commandent pas au même degré l'emploi de phrases courtes. Il y aurait donc plus de place pour les phrases longues dans les genres d'opinion, notamment en chronique. Ainsi, un témoin déclare « aime[r] varier entre le court, le long, [s]'amuser avec tout ça » (P38) et un autre répondant affirme être marqué par une tradition d'écriture qui se caractériserait essentiellement par l'emploi de phrases plus longues, à savoir la littérature proustienne, pourtant condamnée par les enseignants et les manuels d'écriture :

comme je suis proustienne là, j'essaie ... j'essaie d'en couper des fois, parce que ... ça serait ... ça serait ... c'est une erreur d'avoir des phrases trop longues en journalisme, même en chronique, il faut faire attention, moi, moi, ça ... des fois, je me rends compte que je fais ça, puis c'est ... c'est une faute-là, à moins vraiment d'avoir un filon-là qui se suit à merveille, mais on a intérêt à ce que ça soit plus court que long, mais comme je vous dis, euh ... en ... en nouvelle, il y a pas de pardon pour ça ... pour la longueur, en ... en chronique, on a une marge de manœurre (P35)

On reconnait que les témoins accordent une priorité à l'emploi de phrases courtes et simples, mais certains enseignants, et plus encore les chroniqueurs, considèrent l'écart à cette norme idéale de temps à autre comme une pratique souhaitable. Il s'agit d'une position qu'on trouve rarement dans les manuels consultés - seul le guide de Noël présente explicitement la variation de structures syntaxiques comme une stratégie rédactionnelle qui permet d'accrocher les lecteurs et de faciliter la lisibilité des textes, ce qui semble paradoxal compte tenu de la présence dans cet ouvrage, rappelons-le, d'un chapitre consacré à l'importance des phrases courtes:

Variez la longueur des phrases. Vous briserez la monotonie. Une phrase peut compter seulement deux mots. La suivante, une trentaine. La langue française se prête bien aux phrases incomplètes, qui donnent un rythme trépidant à un écrit (Noël, 2009: 191).

\subsection{Clarté et structures lexico-sémantiques}

Les répondants abordent le plus souvent des aspects lexico-sémantiques pour définir la clarté comme un « effort de trouver le mot juste » (P01). Ce dernier est conçu comme un terme parfaitement monosémique « qui correspond le plus possible à la situation qu'on veut décrire » (P10). Les témoins s'appuient donc sur le critère de la précision du vocabulaire et prônent l'idéal de « l'adéquation des mots aux choses » (Paveau et Rosier, 2008 : 277), un peu comme s' « il y a [...] un mot juste pour chaque idée qu'on veut défendre »(P32). En effet, l'idée d'un lien univoque entre la langue et le monde à rapporter représente une fois de plus un argument ancien et constitutif de la clarté 
française qui figure déjà dans les Entretiens d'Ariste et d'Eugène (1671) du père Bouhours à la fin du XVIle siècle (Ludwig et Schwarze, 2012) et qui reste très présent « dans les manuels de savoir-vivre et les guides de correspondance jusqu'à nos jours » (Paveau et Rosier 2008 : 217). Dans cette optique, il est peu étonnant que l'argument du mot juste revienne aussi fréquemment dans la plupart des guides d'écriture journalistique analysés, par exemple dans celui de Ross, où l'auteure estime qu'il n'y a « pas d'information exacte, précise et dépourvue d'ambiguïté en dehors du mot approprié, de l'expression juste » (Ross, 2005 : 119). Dans les entrevues, les témoins reproduisent cette conception du mot juste en ironisant souvent sur l'emploi de mots polysémiques, par exemple des expressions dites passe-partout qui sont considérées comme des termes peu informatifs qui risquent de créer des ambiguïtés dans la communication avec les lecteurs:

il y a des gens qui disent, je suis organisateur d'évènements, j'organise des évènements, tout est un évènement, Monsieur, ça, c'est un évènement, on se rend compte, hein, ma blonde, elle vient de sortir, c'est un évènement, alors je vais dire aux organisateurs, vous organisez quoi (?) des spectacles (?) des ... des spe... des festivals (?) des foires populaires (?) des kermesses (?) je dis à mes étudiants, descendez vers le plus précis, j'ai ... j’ai croisé sur la route un mammifère, oui, l'orignal est un mammifère, mais là, mammifère, alors, c'est ça, c'est un orignal (P24)

Pour les chroniqueurs, la question du mot juste se pose un peu différemment dans les genres journalistiques du commentaire, où il s'agit non seulement de désigner avec exactitude des réalités, mais aussi de les commenter le plus précisément possible en fonction de l'opinion du chroniqueur. De ce point de vue, la recherche du terme propre s'ouvre aussi sur les adjectifs, une catégorie de mots qui serait moins nombreuse dans les genres d'information:

par exemple, je couvrais un budget et [...] tout le monde espérait dans ce budget-là des mesures généreuses envers je sais pas quel groupe et qu'ils ne se ... elles ne s'y trouvent pas, ben, à la nouvelle, on va dire euh ... bon, le ministre n'a pas répondu aux attentes, en chronique, je peux dire ... ah oui, le ministre s'est montré radin, c'est complètement différent, on utilise pas le même vocabulaire, il y a beaucoup ... on va avoir plus de ... d'adjectifs ... mais il faut ... il faut que l'adjectif soit juste, c'est-à-dire il faut pas ... euh ... si j'ai ... si la personne, elle est furieuse ... c'est pas la même chose que si elle est frustrée, il faut utiliser le mot juste, il faut rendre ... il faut être capable de rendre aussi le bon sentiment par rapport à la situation, mais aussi pour rendre notre opinion, il faut utiliser des mots exi... vraiment les mots précis, autant sur le plan factuel que sur le plan émotionnel, descriptif (P36)

9. Décrivant le parler du roi Louis XIV, le père Bouhours juge le mot juste comme simple, naturel et clair : « Ceux qui ont l'honneur de l'approcher admirent avec quelle netteté, et avec quelle justesse il s'exprime. Cet air libre et facile dont nous avons tant parlé entre dans tout ce qu'il dit; tous ses termes sont propres, et bien choisis, quoiqu'ils ne soient point recherchés; toutes ses expressions sont simples et naturelles; mais le tour qu'il leur donne est le plus délicat, et le plus noble du monde » (Bouhours, 1671 dans Hellegouarc'h, 1997 : 29). 
Seuls deux chroniqueurs remettent explicitement en question l'idée d'une correspondance exacte entre les mots et les choses, adéquation qui constitue en effet plutôt l'exception que la règle en langue naturelle (Blank, 2001). Selon eux, «il y a pas qu'un seul mot juste » (P35), mais plusieurs synonymes dont les journalistes peuvent se servir pour décrire le même référent. Cette recherche de synonymes revêtirait également une dimension stylistique qui permettrait aux journalistes de se distinguer les uns aux autres:

je pense que souvent les gens quand ils cherchent ... beaucoup de journalistes, en tout cas, quand ils cherchent le mot juste, c'est toujours le même mot juste, mais il y a des synonymes aussi, tu sais, il faut ... il faut être capable aussi de ... de ... de chercher d'autres mots [...] il y a moyen aussi d'utiliser des synonymes qui vont dire la même chose que ... mais qui sont aussi clairs que ... et qui sont aussi le mot juste, mais ... mais le danger euh ... de dire le mot juste, c'est qu'on ... les gens pensent qu'il y a un seul mot juste là, il y en a ... il y a huit synonymes des fois au mot juste (P35)

Outre la précision du vocabulaire, les témoins invoquent souvent la simplicité des mots pour appuyer leur conception de la clarté lexicale. La définition qu'ils proposent du mot dit simple se fonde tout d'abord sur un critère formel que nous avons déjà présenté à propos de la syntaxe, celui de la longueur des usages. Écrire simplement impliquerait donc de toujours privilégier des « mots courts » (P31), ce qu'un enseignant illustre au moyen de deux paires de mots synonymes:

la clarté, c'est le ... le ... entre deux maux, on dit, il faut choisir le moindre, alors, c'est aussi simple ... au lieu d'écrire le ... le mot le plus clair est toujours le plus court aussi, une question de ... ça se mesure, la lisibilité pour nous, il y a des sociologues de l'information, de la communication, qui ont fait ... qui ont fait des mesures mathématiques de lisibilité, vous avez surement pris connaissance de ça, le nombre de ... de mots avec quatre syllabes et plus, le nombre de mots par phrase, etc., etc., ça donne des quotas ... et euh ... le mot davantage est très français, est très connu, mais on va demander aux ... aux jeunes journalistes d'écrire plus au lieu de davantage, le mot ... euh ... incendie est très bien aussi, moi, je préfère le feu, ça va jusque là, la clarté (P24)

De la même manière, l'utilisation de mots courts est recommandée dans la plupart des manuels, dans lesquels on peut lire, par exemple, qu' «à sens équivalent et à clarté égale, la formulation courte vaut mieux que la longue » (Ross, $2005: 105)$. Il apparait qu'autant les guides d'écriture que les témoins perçoivent les mots courts comme des éléments qui facilitent l'accessibilité des textes, surtout parce que «les gens pourront saisir [des mots courts] aussi en lisant rapidement [...] le journal » (P36). 
Les répondants définissent par ailleurs le mot simple comme un « mot courant » (P24) qui relève d'un registre de langue qui est jugé « accessible aux gens » (P36) et qui permettrait aux journalistes de tenir compte du fait que «c'est pas tout le monde qui a le même vocabulaire » (P36) ${ }^{10}$. En d'autres termes, le souci de clarté et de compréhension s'oppose ici à l'utilisation d'un registre trop soigné qui se compose des usages les plus valorisés, aspect qui est à peine abordé dans la définition des normes communicationnelles proposée par Houdebine $(1988,2002)$. Dans cette optique, les témoins se montrent fermés à l'emploi de termes savants, comme en fait foi l'extrait suivant, dans lequel un enseignant propose de remplacer des mots réputés trop recherchés et complexes par un équivalent qui lui semble plus simple et compréhensible:

clarté, ça veut dire, les mots simples, on ... on ne prétend pas euh ... instruire le lecteur, on ne veut pas instruire le lecteur, c'est très, très, très tentant pour un jeune journaliste de ... d'instruire le lecteur, d'épater le lecteur comme d'épater ses collègues, c'est très tentant, hein de ... par exemple, de dire problématique à la place de problème, ça fait plus universitaire (P24)

L'attitude des auteurs de manuels, comme on pouvait s'y attendre, est unanimement en faveur de l'emploi de mots courants, définis aussi comme des « mots de tous les jours » (Ross, 2005 : 109) qui font partie du « vocabulaire le plus universel possible » (Maltais, 2010 : 19). Ross établit même des liens entre les termes courants et la longueur des mots, un peu comme si les mots fréquemment utilisés étaient presque toujours des termes brefs : "L'emploi de mots courts a donc aussi pour avantage d'aider à respecter la règle du langage usuel. À condition, comme toujours, de ne pas exagérer. Colère est préférable à ire, un archaïsme » (Ross, 2005 : 106).

Malgré ce discours largement véhiculé, plusieurs chroniqueurs déclarent ne pas toujours utiliser des mots courants. Ils demandent plutôt aux lecteurs de faire un certain effort de compréhension, de sorte qu'ils essaient de déduire le sens des mots inconnus à partir du contexte dans lequel ils figurent :

moi, je crois que s'il [le lecteur] le comprend pas tout de suite, il va le comprendre après, il va chercher à le comprendre ou des fois il peut le comprendre dans le contexte, c'est un peu quand on lit une langue étrangère, tu lis une phrase, il y a ... il y a un mot que tu comprends pas, mais tu es capable de resituer ... tu es capable de deviner plus ou moins en fonction du contexte, c'est sûr que c'est moins précis pour le lecteur, mais en même temps, la facilité ... on est dans une époque de facilité là, on passe not... nos ... nos semaines dans des ... dans des environnements ... euh ... culturels où la facilité a été érigée en ... en dogme ... ça fait que ... si on peut se garder des espaces ... euh ... où il y a peut-être un peu plus de profondeur, où il y a de ... de ... de la culture fine euh ... pourquoi s'en priver (?) (P34)

10. La politique linguistique de la Société Radio-Canada définit aussi les termes du registre courant comme «simples » (Radio-Canada, 2004 : 22). Ainsi, tant en contexte de presse écrite que de presse radiophonique et télévisuelle, les registres de langue tendent à être définis « non pas en fonction de la situation de communication, mais en fonction de leurs caractéristiques 'pseudo-linguistiques' » (Remysen, 2010 : 132). 
Le chroniqueur conçoit le recours à des mots rares comme une stratégie rédactionnelle qui lui permet de se distinguer au sein du marché journalistique et de mettre en valeur ses propres prises de position. Dans la même veine, un autre chroniqueur prétend « aime[r] placer un ou deux mots un peu plus rares dans un texte» (P38). Pour lui, l'idée que les lecteurs doivent « ouvrir leur dictionnaire pour trouver ce terme-là » (P38) revêt plutôt un aspect ludique et interactif qui devrait rendre la lecture de ses textes plus intéressante:

j'aime ça, j'aime ça, c'est un plaisir là, et ... et euh ... et ici, c'est presque ... comment on dit ... c'est un running gag, mais ... ah, tu sais, encore tu m'as fait ouvrir mon dictionnaire pour trouver ce ... ce terme-là, tu sais, c'est un mot qu'on voit pas souvent, moi, j'aime ça des fois aussi, obliger le lecteur à ouvrir son dictionnaire, parce que ce terme-là ... peut-être que tu l'as pas vu souvent, puis ... est-ce que ça veut vraiment dire ça (?) (P38)

Enfin, un chroniqueur déclare se donner parfois pour tâche de faire sortir de l'oubli des mots à fréquence basse pour les faire connaitre aux lecteurs. Dans la citation suivante, ce témoin se présente en quelque sorte comme un sauveteur de mots en voie de disparition :

[il faut pas] se priver de placer des mots euh ... rares, moi, j'aime ça, placer des mots des fois qu'on a pas l'habitude d'entendre, que je ... je considère ... je fais ça d'ailleurs en considérant un peu sauver ce mot-là en disant, mais tiens, il sera pas ... il sera pas aux oubliettes, sortir les mots des oubliettes, c'est bon ça (P34)

La volonté de sauver des mots constitue de nouveau un sujet fréquent dans le discours sur la langue française (Paveau et Rosier, 2008 : 222). Néanmoins, dans le cas présent, il est étonnant de voir que cet argument est avancé par un professionnel des médias de masse, dans lesquels l'intention de conserver des mots rares semble aller à l'encontre du besoin « d'être clair et compris, d'être efficace pour leurs lecteurs » (Houdebine, 1988 : 140). On conclut donc que les chroniqueurs peuvent ne pas toujours vouloir adapter leurs usages en fonction des normes communicationnelles, ce qui en fait un groupe de journalistes à part qui se distinguent dans une certaine mesure des journalistes de l'information qui doivent rendre leurs textes compréhensibles à tout prix (Meier, à paraitre). Cette observation nous permet de poser l'hypothèse selon laquelle le respect (ou non-respect) des normes communicationnelles peut varier selon les différents genres journalistiques. 


\subsection{Clarté et structure textuelle}

Les répondants considèrent parfois la structure textuelle comme une autre composante de la clarté journalistique. Ainsi, il conviendrait aussi de réfléchir à l’ordre logico-sémantique dans lequel les journalistes présentent une information aux lecteurs. Les témoins se réfèrent ici plus ou moins explicitement à la cohésion et à la cohérence en tant que principes généraux de l'organisation textuelle $\mathrm{e}^{11}$. On observe alors un chevauchement entre normes textuelles et normes communicationnelles.

De ce point de vue, les enseignants affirment l'importance de « hiérarchiser et ordonnancer» (P11) les éléments principaux d'un contenu rédactionnel dans de « bons regroupements qu'on doit sentir » (P11), c'est-à-dire en fonction d'un plan de texte où

les idées progressent de manière ... où il y a une linéarité dans les idées et donc ... qui permet aux lecteurs de suivre un propos sans se perdre dans des méandres, donc, c'est important, cette ... cette clarté de structure (P04)

Dans la même veine, un autre enseignant souligne que la clarté de l'ordre des idées se fonderait généralement sur « quelque chose qui est transversal, qui est le fil conducteur, qui souvent prend la forme d'un récit » (P11). Autrement dit, la clarté relèverait surtout de l'agencement cohérent des différentes parties d'un texte, dont la succession devrait suivre de préférence le schéma d'une macrostructure narrative. À l'inverse, ce témoin considère en toute logique qu'une organisation de contenus rédactionnels peu cohérente donnerait forcément lieu à des textes

où on [les lecteurs] ... on n'a pas envie de suivre, c'est pas intéressant parce que souvent, c'est qu'il y a pas de fil de conducteur, on n'a pas de ... on n'a pas de récit et ce qui fait souvent la clarté aussi, c'est qu'on puisse suivre un fil et le fil va être dans bien des cas, dans le fond, une sorte de récit, qu'il y a pas forcément à mettre des personnages en ... en ... en ... en contexte, mais qui va permettre en fait aux lecteurs de suivre l'histoire et de voir qu'il y a un début, un milieu, une fin (P11)

On observe une argumentation semblable dans les commentaires des chroniqueurs, qui soutiennent que «la clarté, c'est [...] un déroulement logique du récit parce qu'on récite [...] on raconte une histoire » (P19). Ils estiment que la clarté structurelle est particulièrement importante dans la chronique, où il s'agit de développer une argumentation bien fondée pour défendre son opinion personnelle devant les lecteurs. L'importance accordée à ce type de clarté est d'autant plus compréhensible que la chronique ne connait pas un plan de texte prédéfini (Meier, à paraitre) et que la

11. Selon Adam, la notion de cohésion désigne «l'ensemble des moyens linguistiques qui assurent les liens intra- et interphrastiques permettant à un énoncé oral et écrit d'apparaître comme un texte » (2002: 99). Inversement, « [d] es liens d'ordre logico-sémantiques [sont] plutôt à déduire pour construire la cohérence, [qui] n'apparait pas comme une propriété strictement linguistique des textes. Elle résulte d'un jugement qui prend appui sur la connaissance de la situation et les savoirs lexico-encyclopédiques des sujets » (Adam, 2002 : 99). 
particularité du genre provient justement de la créativité de l'enchainement des idées présentées dans le texte, comme le montre l'extrait ci-après:

je trouve dans un texte argumentatif ou dans un ... dans une chronique qui est plus euh ... de type éditorial, à avoir une structure de pensée qui est claire, limpide, et où le ... le lecteur va rapidement comprendre de quoi on parle, de quoi il s'agit, où on s'en va, c'est quoi notre point de vue, et ... et ça ... euh ... je trouve que ... c'est un peu ce à quoi j'aspire quand ... quand j'écris une chronique ... j'y arrive malheureusement pas souvent, je ... je suis rarement satisfait de ... de ce que je fais, mais je ... j'essaie de faire ça, donc, effectivement la clarté, c'est ... c'est aussi primordial (P38)

Plusieurs témoins estiment que les agents du discours normatif sur les pratiques communicationnelles mettent trop l'accent sur la conceptualisation de la clarté comme phénomène syntaxique et lexical, omettant ainsi souvent le fait que la structure du texte contribue elle aussi à une présentation claire et transparente de l'information. Comme on peut le voir dans la citation suivante, un enseignant part plutôt de l'idée selon laquelle la clarté constitue le résultat d'un « ensemble de choses » (P11) qui, en réalité, s'emmêlent et qui sont articulées en une hiérarchie, où la clarté textuelle semble avoir plus de poids que la clarté syntaxique et lexicale :

je pense qu'on a trop tendance à voir la clarté dans ce qu'on appelle le lexico-syntaxique là, le lexique et la syntaxe, c'est-à-dire uniquement ... on parle beaucoup de clarté pour le choix des mots et c'est vrai que ça en fait partie, et la structure de la phrase-là ... la syntaxe, mais à mon avis, c'est quelque chose d'important, mais c'est pas ... uniquement ça, je crois que la ... la clarté d'un texte, c'est une clarté qui peut être dans la structure macro du texte, c'est-à-dire que je pense que le ... le ... dès le départ de la sélection de l'information et dès le choix des regroupements de l'information, de la hiérarchisation de l'information et de l'ordonnancement de l'information, je pense que c'est ... chacun de ces éléments-là fait en sorte qu'on arrivera ou non à la clarté de ... d'un message (P11)

Écrire clairement serait donc fondé à priori sur une démarche de compréhension par rapport aux contenus rédactionnels à transmettre. Cette citation fait écho aux propos d'autres témoins selon qui la clarté passe aussi par « un travail [et] une gymnastique constante » (P02) et non seulement par un ensemble de « recette[s] magique[s] » (P02, P10, P25). En d'autres termes, la clarté ne se définit pas exclusivement en fonction d'un éventail de choix lexico-syntaxiques décrits dans les manuels. 
Contrairement à ce que les témoins laissent entendre, les guides d'écriture n'écartent pas complètement la dimension textuelle de leurs réflexions, même s'ils instaurent plutôt des règles qui visent la clarté syntaxique et lexicale ${ }^{12}$. Prenons par exemple le manuel de Ross : « Une fois qu'on a une idée claire de ce qu'on veut dire, on cherchera à bien le dire. Cela concerne le vocabulaire et la syntaxe. Et aussi l'organisation globale du texte, le plan »(Ross, 2005 : 101). Par ailleurs, il est significatif que le discours des manuels montre à nouveau de fortes similitudes avec celui des témoins, notamment en ce qui concerne la nécessité d'établir une connexion cohérente entre les différentes composantes d'une information, et ce, sous forme d'un fil conducteur:

Plus un texte est structuré de façon efficace, plus il a de chances d'être intelligible. Il y a toutefois une limite à la 'réduction' du propos. L'intelligibilité (ou la clarté) vous impose de faire tous les liens requis, tous les rappels utiles, bref, de fournir à l'ensemble de vos lecteurs tous les éléments d'information essentiels à la bonne compréhension (Sormany, 2011 : 101).

\section{Conclusion}

L'analyse du corpus a illustré que la clarté constitue un principe fondamental de l'écriture journalistique que les témoins perçoivent généralement comme un concept polymorphe qui peut comporter des éléments certes très diversifiés, mais qui sont souvent liés les uns aux autres. Notre analyse a par ailleurs révélé que ces éléments ne sont pas d'égale valeur dans l'imaginaire linguistique des répondants, mais organisés de façon hiérarchisée. S’il est vrai que, sur le plan quantitatif, les témoins mentionnent le plus souvent des aspects syntaxiques et, plus encore, des aspects lexico-sémantiques, ils attribuent néanmoins une grande importance à la clarté de la structure textuelle. On constate donc que les arguments traditionnels du discours sur la clarté française, comme ceux de l'ordre naturel ou du mot juste, sont bien présents dans les commentaires des témoins, d'autant plus qu'il s'agit de paradigmes rédactionnels véhiculés par les manuels analysés, qui semblent avoir une certaine influence sur le discours des répondants. Cependant, ces vieux arguments tendent à céder la place à des éléments plus nouveaux, qui concernent non seulement le choix de registre, mais aussi et surtout la cohésion et la cohérence textuelles. Cette observation rappelle celle d'un enseignant québécois, qui constate à propos du Rapport du Groupe de travail sur la réforme du curriculum (1997) que « la cohérence est la nouvelle forme du mythe de la clarté de la langue » (Lecavalier, 1998 cité dans Paveau et Rosier, 2008 : 174).

La hiérarchisation des différentes composantes de la clarté journalistique s'exprime par ailleurs dans la façon dont les témoins tolèrent des usages qui ne sont pas immédiatement saisissables par les lecteurs. Si le respect de la structure textuelle est généralement considéré comme un préalable indispensable à la compréhension d'articles, les répondants semblent s'accorder une certaine

12. Tous les manuels analysés consacrent plusieurs sections à la structure des textes journalistiques. Cependant, la question des rapports entre la clarté de l'écriture journalistique et la structure textuelle n'y est généralement pas évoquée et fait plutôt l'objet des sections qui traitent de plus près des particularités du style fonctionnel journalistique. 
marge de manœuvre sur le plan syntaxique et, de façon plus importante encore, sur le plan lexical. Ainsi, plus une composante de clarté réfère à des éléments complexes, plus elle semble constituer un repère normatif à respecter. Dans cette perspective, on comprend pourquoi plusieurs témoins déclarent accepter l'utilisation de mots peu courants à condition que la structure textuelle permette d'assurer la compréhension du message.

Les témoins invoquent essentiellement deux arguments pour légitimer la transgression des normes communicationnelles établies, aspects qui semblent étroitement liés aux évolutions que l'on observe actuellement en rédaction journalistique. D'abord, les répondants s'appuient sur des arguments d'ordre stylistique pour souligner qu'une écriture plus rythmée pourrait parfois mieux assurer la communication avec les lecteurs qu'une écriture uniforme qui suit strictement le modèle canonique de la phrase. Cette observation témoigne d'une certaine ouverture à l'endroit d'une écriture de presse plus individualisée, ce que Charron et de Bonville (2004 : 206) considèrent comme une des principales caractéristiques du journalisme de communication. Ensuite, certains chroniqueurs affirment parfois vouloir délibérément rendre leur message difficile à déchiffrer de sorte que les lecteurs doivent faire plus d'efforts pour reconstruire le sens d'un message. II apparait que ces chroniqueurs attribuent aux lecteurs un rôle plus actif, voire participatif, dans la communication d'informations, ce que Grevisse estime être, rappelons-le, le reflet d'une « individualisation du média de masse » (2008: 213) qui rend compte du poids accru des moyens de communication dits nouveaux, permettant à l'individu « de construire son propre récit, protéiforme, de la réalité » (Grevisse, 2008 : 213). La clarté ne constituerait donc plus à priori un impératif rédactionnel absolu qui sert à joindre le plus grand nombre, mais elle serait plutôt considérée comme le résultat d'un processus de négociation de sens entre le journaliste et le lecteur. 


\section{Références}

Adam, Jean-Michel (2002), « Cohérence», dans Patrick Charaudeau et Dominique Maingueneau (dir.), Dictionnaire d'analyse du discours, Paris, Éditions du Seuil, p. 99-100.

Bell, Allan (1991), The Language of News Media, Oxford (Royaume-Uni)/Cambridge (États-Unis), Blackwell.

Blank, Andreas (2001), Einführung in die lexikalische Semantik für Romanisten, Tübingen, Max Niemeyer Verlag.

Brin, Colette, Jean Charron et Jean de Bonville (2004), "Introduction », dans Colette Brin, Jean Charron et Jean de Bonville (dir.), Nature et transformation du journalisme. Théorie et recherches empiriques, Québec, Les Presses de l'Université Laval, p. 1-31.

Burger, Harald (2000), "Textsorten in den Massenmedien », dans Klaus Brinker, Gerd Antos, Wolfgang Heinemann et Sven Sager (dir.), Text- und Gesprächslinguistik. Ein internationales Handbuch zeitgenössischer Forschung, vol. 1, Berlin/New York, Walter de Gruyter, p. 614-628.

Burger, Harald (2005), Mediensprache. Eine Einführung in Sprache und Kommunikationsformen der Massenmedien, 3e éd., Berlin/New York, Walter de Gruyter. [1're éd., 1984.]

Charaudeau, Patrick (1988), «Une théorie des sujets du langage », Modèles linguistiques, n 10, p. 6778.

Charron, Jean et Jean de Bonville (2004), «Typologie historique des pratiques journalistiques », dans Colette Brin, Jean Charron et Jean de Bonville (dir.), Nature et transformation du journalisme. Théorie et recherches empiriques, Québec, Les Presses de l'Université Laval, p. 141-217.

Eroms, Hans-Werner (2008), Stil und Stilistik. Eine Einführung, Berlin, Erich Schmidt Verlag.

Fairclough, Norman (1995), Media discourse, London, Hodder Education.

Grevisse, Benoît (2008), Écritures journalistiques. Stratégies rédactionnelles, multimédia et journalisme narratif, Bruxelles, De Boeck.

Hellegouarc'h, Jacqueline (1997), L'art de la conversation. Anthologie, Paris, Dunod.

Houdebine, Anne-Marie (1983), «Sur les traces de l'imaginaire linguistique? », dans Verena Aebischer et Claire Forel (dir.), Parlers masculins, parlers féminins?, Neuchâtel, Delachaux/Niestlé, p. 105139.

Houdebine, Anne-Marie et Corinne Baudelot (1985), "L'imaginaire linguistique dans la communication mass-médiatique », dans Patrick Charaudeau (dir.), Médias et enseignement. Actes du colloque AUPELF, Sitges, avril 1984, Paris, Didier Érudition, p. 58-64.

Houdebine, Anne-Marie (1988), «Elle parle français la presse ! ou La Belle au Bois Dormant des analyses de discours », dans Patrick Charaudeau (dir.), La Presse. Produit. Production. Réception, Paris, Didier Érudition, p. 131-149. 
Houdebine-Gravaud, Anne-Marie (2002), «L'Imaginaire linguistique : un niveau d'analyse et un point de vue théorique », dans Anne-Marie Houdebine-Gravaud (dir.), L'imaginaire linguistique, Paris, L'Harmattan, p. 9-21.

Lavoinne, Yves (1991), «Le journalisme saisi par la communication », dans Marc Martin (dir.), Histoire et médias. Journalisme et journalistes français, 1950-1990, Paris, Albin Michel, p. 161-173.

Lebsanft, Franz (2001), «Sprache und Massenkommunikation », dans Günter Holtus, Michael Metzeltin et Christian Schmitt (dir.), Lexikon der Romanistischen Linguistik, vol. 1, n² 2, Tübingen, Max Niemeyer, p. 292-304.

Lecavalier, Jacques (1998), «Cohérence ou pertinence? », Correspondance, vol. 4, n² 2, disponsible sur http://correspo.ccdmd.qc.ca/Corr4-2/Pertin.html. [Page consultée le 15 juillet 2016.]

Ludwig, Ralph et Sabine Schwarze (2012), «Ein erneuter Blick auf Entwicklungen der französischen Sprachkultur : zur Vernetzung von sprachlicher Normierung und literarischer Kanonisierung im 18. und frühen 19. Jahrhundert », Romanistisches Jahrbuch, vol. 62, n 1, p. 98-136.

Maltais, Robert (2010), L'écriture journalistique sous toutes ses formes, Montréal, Presses de l'Université de Montréal.

Meier, Franz (2016). La perception des normes textuelles, communicationnelles et linguistiques en écriture journalistique. Une contribution à l'étude de la conscience linguistique des professionnels des médias écrits québécois, thèse de doctorat, Augsburg, Universität Augsburg.

Meier, Franz (à paraitre), « Normes textuelles et construction d'une identité professionnelle : le cas des chroniqueurs de la presse écrite québécoise », Communication et professionnalisation, n̊ 4 .

Noël, André (2009), Le style. Conseils pour écrire de façon claire et vivante, Montréal, Éditions La Presse.

Paveau, Marie-Anne et Laurence Rosier (2008), La langue française. Passions et polémiques, Paris, Vuibert.

Radio-Canada (2004), La qualité du français à Radio-Canada : principes directeurs, document non publié.

Riegel, Martin, Jean-Christophe Pellat et René Rioul (2008), Grammaire méthodique du français, $5^{\circledR}$ éd., Paris, Presses Universitaires de France. [1'e éd., 1994.]

Rivarol, Antoine (1784), De l'universalité de la langue françoise, Berlin/Paris, Bailly \& Dessenne. [édition critique par Marcel Hervier (1929), sous le titre Discours sur l'universalité de la langue française, Paris, Delagrave.]

Remysen, Wim (2010), « La politique linguistique des médias au Québec et en Flandre : de quelle conception de la langue est-il question? ", dans Diane Vincent et Wim Remysen (dir.), Hétérogénéité et homogénéité dans les pratiques langagières. Mélanges offerts à Denise Deshaies, Québec, Les Presses de l'Université Laval, p. 115-150. 
Ross, Line (2005), L'écriture de presse. L'art d'informer, 2e éd., Montréal, Gaëtan Morin. [1re éd., 1990.]

Sanders, Willy (1977), Linguistische Stilistik. Grundzüge der Stilanalyse sprachlicher Kommunikation, Göttingen, Vandenhoeck \& Ruprecht.

Sormany, Pierre (2011), Le métier de journaliste, 3 ee éd., Montréal, Boréal. [1e éd., 1990.]

Swiggers, Pierre (1987), «A l’ombre de la clarté française », Langue française, vol. 75, nº 1, p. 5-21.

Swiggers, Pierre (2010), "La clarté du français : examen d'un 'idéologème' », Zeitschrift für Romanische Philologie, vol. 126, p. 443-459.

Thompson, John (1995), The Media and Modernity : A Social Theory of the Media, Cambridge, Polity Press.

Weinrich, Harald (1961), "Die clarté der französischen Sprache und die Klarheit der Franzosen », Zeitschrift für Romanische Philologie, vol. 77, p. 528-544.

Yaguello, Marina (2008), Catalogue des idées reçues sur la langue, $3^{e}$ éd., Paris, Éditions du Seuil. [1'éd., 1988.] 